\title{
Legal Medicine Evaluation of a Laboratory Error at the Blood Transfusion Center of Iran: A Case Report
}

Naimeh Farhidnia, Azadeh Memarian*and Fardin Fallah

Department of Forensic Medicine, Iran University of Medical Sciences, Tehran, Iran

*Corresponding author: Azadeh Memarian, Department of Forensic Medicine, Iran University of Medical Sciences, Tehran, Iran, Tel: 98-21-66551201; E-mail: azade.memarian@gmail.com

Received date: May 18, 2016, Accepted date: Jul 02, 2016, Publication date: Jul 05, 2016

Copyright: (c) 2016 Farhidnia N, et al. This is an open-access article distributed under the terms of the Creative Commons Attribution License, which permits unrestricted use, distribution, and reproduction in any medium, provided the original author and source are credited.

\begin{abstract}
Wrong blood transfusion complication is one of the medical malpractices in Iran. A 57-year-old female patient was admitted with a diagnosis of discopathy that required an operation. Her Blood groups was $O$ negative, but no one was aware. After initial cross-match blood group A was injected. Necrosis and edema with blisters and severe pain on the injected hand was seen after three to four hours. Unfortunately complications occurred due to receiving wrong blood transfusion. The patient was treated with high-dose corticosteroids and fasciotomy. Hematoma was drained. Hemodialysis was performed because of increased creatinine. After two months, the patient discharged with good general appearance. The patient sued the hospital. The lab technician was found guilty and was sentenced to pay $20 \%$ of the full wergild.
\end{abstract}

Keywords: Laboratory; Blood transfusion; Error; Iran

\section{Introduction}

Transfusion of blood saves one's life. An error in blood transfusion, at the same time, takes life [1]. Hospital negligence can lead to blood transfusion errors that result in injuries and even fatalities. Blood transfusion errors include: mislabeled blood; the wrong patient receiving a blood transfusion; the patient receiving the wrong blood type; and hospital staff failing to respond to the signs and symptoms of a blood transfusion error [2]. According to studies, the risk of errors in blood transfusion was about 1 per 12000 to 19000 cases in the United States and the rate of fatal transfusion was 1 per 600000 to 800000 $[3,4]$.

Red Blood Count (RBC) incompatibility reactions consist of two parts: hemolytic reactions with presentations including bilirubinemia, renal failure, hemoglobinuria, elevated lactate dehydrogenase, positive direct Coombs test shock, and Adult Respiratory Distress Syndrome (ARDS). These occur as a result of massive intravascular hemolysis, which are because of presentation of complement fixing antibodies in the recipient's serum; delayed transfusion reaction, that is brought about by IgG antibodies that do not fix the complement. The signs include fever, raised bilirubin, decreased hemoglobin, jaundice, and hemoglobinuria that are related to extravascular hemolysis $[5,6]$.

Studies have shown several ways to reduce the number of blood transfusion errors, for example a unit blood sample should be confirmed by two individuals, using advanced technological systems, with computerized barcode-based coupling of the patient ID band with blood product labels along with Radiofrequency Identification technology (RFID) $[7,8]$, and locating locking devices on the blood product ports with single codes for the patient and the product $[7,8]$.

Wrong blood transfusion complication is one of the medical malpractices found in Iranian hospitals. In this study, a female patient was admitted with a diagnosis of discopathy that needed an operation. A wrong blood group was injected for her and unfortunately complications occurred because of having received the wrong blood type. In fact, this study evaluated a medical malpractice and an approach in dealing with it according to the Iranian penal code.

\section{Case Presentation}

A 57-year-old female patient was admitted with a diagnosis of Discopathy that needed an operation. Blood group and Intensive Care Unit (ICU) was reserved in the morning of the surgery and then the patient was transferred to the operating room. The patient had a history of diabetes and blood pressure. Under anesthesia, she required blood transfusion. Her blood group was $\mathrm{O}$ negative, but no one was aware. After initial cross-match, blood group A was injected. After the operation, the patient was transferred to the ICU with a Glasgow Coma Scale (GCS) of 8 . The consciousness of the patient gradually increased. Necrosis and edema with blisters and severe pain on the injected hand was seen after three to four hours (Figure 1). Rheumatology, ultrasonic and surgical consultation was requested. Hematoma was reported. Because of drop in hemoglobin, blood transfusion was required. Cross-matching was performed again and blood group $\mathrm{O}$ was determined and injected. Delayed transfusion reaction occurred because of wrong cross-match and receiving the wrong blood type. The patient was treated with high-dose of corticosteroids and fasciotomy. Hematoma was drained. Hemodialysis was performed because of increased creatinine. Finally, after two months, the patient was discharged with good general appearance. The patient sued the hospital. The patient was not aware of his blood group and unfortunately nobody took history from the patient and the lab technician was reported the wrong result for cross-match. Due to the severe bleeding during the surgery transfusion was performed. The hemoglobin level of patient was normal in patient's report but the coagulation condition of patients wasn't evaluated before surgery. According to the evidence patient was suffered from coagulation disorder and therefore malpractice was done in evaluation of preoperative coagulation factors. The prescribed antibiotic was ceftriaxone and eculizumab is not presented in that geographical area. 
The lab technician was found guilty too and was sentenced to pay $20 \%$ of the full wergild.

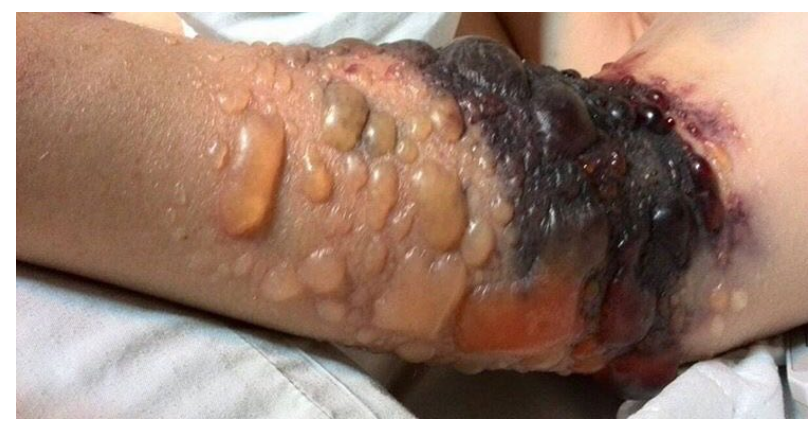

Figure 1: Necrosis and Edema with Blisters and Severe Pain on the Injected Hand.

\section{Discussion}

Transfusion errors are incidences that are regrettably too common, and prevention of these mistakes is a significant medical concern $[9,10]$. Transfusion of incorrect blood products with the following acute hemolytic reaction was the second cause of death from transfusion, between years 2005 and 2009, and one of the most preventable reasons of transfusion-related morbidity $[3,11]$.

Our report demonstrates errors made by lab technicians. Medical personnel are legally liable for their professional errors. These errors are referred to as medical malpractice, and are divided to four groups of carelessness, imprudence, misconduct, and disregard for governmental rules and medical principals. Carelessness is when a specific medical procedure should not have been performed on a particular patient but is done on the patient. Imprudence is when a specific procedure should have been performed for a particular patient but is not done on the patient [12]. The patient receiving the wrong blood type is considered imprudence.

According to the article 448 of the Iranian penal code, the wergild is a specific amount determined by the sacred law for an unintentional crime against life, limb or benefit, or an intentional crime with no punishment (for whatever reason). Article 145 of the Iranian penal code states; the realization of an unintentional crime is subject to the proof of the offender's guilt. For unintentional crimes, including manslaughter and pure mistake, the rules of the book of retributions and wergild shall apply. Guilt includes carelessness and imprudence. Carelessness, negligence, inaptitude, and disrespect for the governmental orders and such like, depending on the case, are considered examples of carelessness or imprudence [13].

In conclusion, the patient was discharged from the hospital and sued the hospital. The lab technician was found guilty for mistake in cross-match test results and was sentenced to pay $20 \%$ of the full wergild according to the Iranian penal law.

\section{References}

1. http://www.asrn.org/journal-nursing/285-blood-transfusion-errorprevention-nurses-role.html

2. https://www.hg.org/article.asp?id=28136

3. Linden JV, Paul B, Dressler KP (1992) A report of 104 transfusion errors in New York State. Transfusion 32: 601-606.

4. Linden JV, Wagner K, Voytovich AE, Sheehan J (2000) Transfusion errors in New York State: an analysis of 10 years' experience. Transfusion 40: 1207-1213.

5. Nuttall GA, Stubbs JR, Oliver WC Jr (2014) Transfusion errors: causes, incidence, and strategies for prevention. Curr Opin Anaesthesiol 27: 657-659.

6. Behnia M (2007) Complications of Blood Transfusion in Critical Situation: A Concise Overview. Tanaffos 6: 15-19.

7. Heddle NM, Fung M, Hervig T, Szczepiorkowski ZM, Torretta L, et al. (2012) Challenges and opportunities to prevent transfusion errors: a Qualitative Evaluation for Safer Transfusion (QUEST). Transfusion 52: 1687-1695.

8. Coustasse A, Cunningham B, Deslich S, Willson E, Meadows P (2015) Benefits and Barriers of Implementation and Utilization of RadioFrequency Identification (RFID) Systems in Transfusion Medicine. Perspect Health Inf Manag 12: 1d.

9. Adibi H, Khalesi N, Ravaghi H, Jafari M, Jeddian AR (2012) Root-cause analysis of a potentially sentinel transfusion event: lessons for improvement of patient safety. Acta Med Iran 50: 624-631.

10. Maskens C, Downie H, Wendt A, Lima A, Merkley L, et al. (2014) Hospital-based transfusion error tracking from 2005 to 2010: identifying the key errors threatening patient transfusion safety. Transfusion 54: 66-73.

11. Sazama K (1990) Reports of 355 transfusion-associated deaths: 1976 through 1985. Transfusion 30: 583-590.

12. Khoshhal M, Vafaee F, Khoshhal M (2013) Rules Governing the Liability of Dentists in Iran. IJFM 19: 227-232.

13. http://www.ghanoonbaz.com/anavin/kaifary/mojazat.htm 